\section{Keratocyte density and size in conscious humans by digital image analysis of confocal images}

\begin{abstract}
Purpose Confocal microscopy can give images of high magnification and resolution in undisturbed living tissue. It provides new information about the cellular structure of the cornea. Our aim was to measure the density, size and distribution of keratocytes.

Methods Healthy cornea in four subjects was examined using tandem scanning confocal microscopy. Methods for digital analysis of images were developed.

Results Keratocyte density in confocal crosssections was greatest immediately under Bowman's membrane (maximum 800 cells/ $\mathrm{mm}^{2}$ ) and decreased sharply towards posterior cornea (minimum 65 cells $/ \mathrm{mm}^{2}$ ). Crosssectional cell size ranged from 78 to $211 \mu \mathrm{m}^{2}$, but did not correlate with depth in the tissue. Conclusions Results are consistent with those of earlier work using histological and biochemical techniques in isolated tissue. The methods we have developed enable studies of ongoing processes in conscious humans and can be used to examine diseased tissue as well as the response to injury.
\end{abstract}

Key words Confocal microscopy, Cornea, Corneal stroma, Image analysis, Keratocytes

Confocal microscopy can give images of high magnification, resolution and contrast throughout the cornea in conscious co-operative human subjects. ${ }^{1}$ Conventional histological techniques of sectioning, fixation and staining are not required to identify structures. It provides the opportunity to examine ongoing physiological and pathological processes in undisturbed living tissue.

Video recordings of confocal microscopy can give several thousand different images in each subject. Imaging of such large amounts of information has not been possible in the past and new techniques are required to extract usable data. We developed computerised methods for analysing cellular structure in the stroma of conscious humans. These gave independent automated measurements of the density and size of keratocytes. The technique and results in normal subjects are presented in this paper. Changes during wound healing will be presented in a later publication.

Klyce and Beuerman ${ }^{2}$ examined keratocyte structure using electron microscopy. Long cytoplasmic processes tapering to about $1 \mu \mathrm{m}$ in diameter were described. In our work such processes were not seen. They were probably too fine to be resolved by the optical system. Thus confocal images of keratocytes show only a portion of the cell, perhaps just the nucleus or the nucleus and part of the cell body. In this paper, keratocytes imaged by confocal microscopy are referred to as 'cells', as the exact portion seen in images is unknown.

Keratocytes function to maintain the collagen fibres and extracellular matrix of the corneal stroma. $^{2}$ Earlier work concerning their density and distribution was done using conventional microscopy or biochemical methods in excised tissue. ${ }^{3-6}$ Petroll et al. ${ }^{7}$ used a laser scanning confocal microscope to examine isolated rabbit cornea. Images were digitised for computer analysis, but each cell was marked manually to enable measurement of their density.

\section{Materials and methods}

\section{Equipment}

A tandem scanning confocal microscope (Tandem Scanning Corporation, Reston, Virginia, USA) was used with $100 \mathrm{~W}$ mercury arc lamp and $\times 24$ objective (numerical aperture 0.6 ). The microscope has been described by Cavanagh et al. ${ }^{1}$ The position of the camera and final condensing lens was adjusted so that as much as possible of the image fell on the photocathode of the camera, thus increasing the field of view $(630 \times 420 \mu \mathrm{m})$. Images were recorded using a DAGE VT1000 silicon intensified tube camera (SIT) and Panasonic S-VHS recorder (AG-7355). Optical sections are curved with the apex away from the eye under
J.I. Pryda

M.G. Kerr Muir

M.C. Corbett

J. Marshall

St Thomas' Hospital and UMDS

London SE1 7EH, UK

J.I. Prydal

Department of

Ophthalmology (Box 41)

Addenbrooke's Hospital

Hills Road

Cambridge CB2 2QQ, UK

Tel: +44 (0)1223464969

Fax: +44 (0)1223 778282

e-mail:prydal@

elmc demon co.uk

F. Franc

Institute of Physics

Department of Applied

Optics

Academy of Sciences of

Czech Republic

Prague

Czech Republic

P.N. Dilly

St George's Hospital

Medical School

Department of Anatomy London SW17 ORE, UK

We would like to thank the Iris Fund and Special Trustees of St Thomas' Hospital London for their generous support. M.C.C. holds the William's

Fellowship for Medical and Scientific Research 
examination. The radius of curvature is similar to that of the cornea (J. Hill, Tandem Scanning Corporation, personal communication). Petroll et al. ${ }^{8}$ determined axial resolution of optical sections to be $9 \mu \mathrm{m}$.

A special feature of this confocal microscope is that the plane of focus can be advanced through the cornea without movement of the objective or object. A separate unit (Oriel controller 180011) controls motor-driven lenses within the objective. It can be set to move the focal plane in or out of the tissue, either continuously or in specified steps. A readout gives the distance moved by lenses in the objective. Using computer models of the optics, the manufacturer has provided a polynomial equation converting this reading to the distance moved by the optical section through the stroma.

\section{Procedure}

Four subjects ( 2 men, 2 women; age 23-39 years) were fully informed of the nature of the procedure and gave their consent to be examined. The cornea was anaesthetised with $0.4 \%$ oxybuprocaine hydrochloride and the subject's head supported by chin and forehead rests. Subjects were instructed to fixate a small light. Immersion fluid was $2 \%$ methylcellulose. After contact between objective and eye, the focal plane was advanced and the position of the microscope adjusted until images of superficial epithelium were circular. Optical sections were then tangential to the cell surface. The plane of focus was advanced into the most superficial layer of keratocytes and then in steps of $50 \mu \mathrm{m}$ ( $25 \mu \mathrm{m}$ in one of the subjects). Pauses for about $5 \mathrm{~s}$ were recorded at each level. Depth readout was recorded verbally on the audio track. When the endothelial surface was reached fine adjustments were made to image the layer of keratocytes just superficial to Descemet's membrane.

\section{Digitising of images}

Single fields of video recordings were digitised to a resolution of $768 \times 512$ pixels and 256 grey levels (Overlay Frame Grabber, Imaging Technology, Woburn, Massachusetts, USA). Ten fields at each level were digitised for all subjects, giving a total of 100-200 images per subject. The only criteria used in selecting images were that they should not be blurred or show stromal nerves. The presence of nerves complicated computer analysis and made measurements unreliable.

\section{Problems in analysis of images}

A sample image is shown in Fig. 1. Keratocytes appeared as bright objects against a dimmer background. In our early attempts at processing, binary images (two brightness levels) were produced from grey-scale images (256 brightness levels). Pixels with values greater than a threshold level were set to 1 and those with smaller values to 0 .

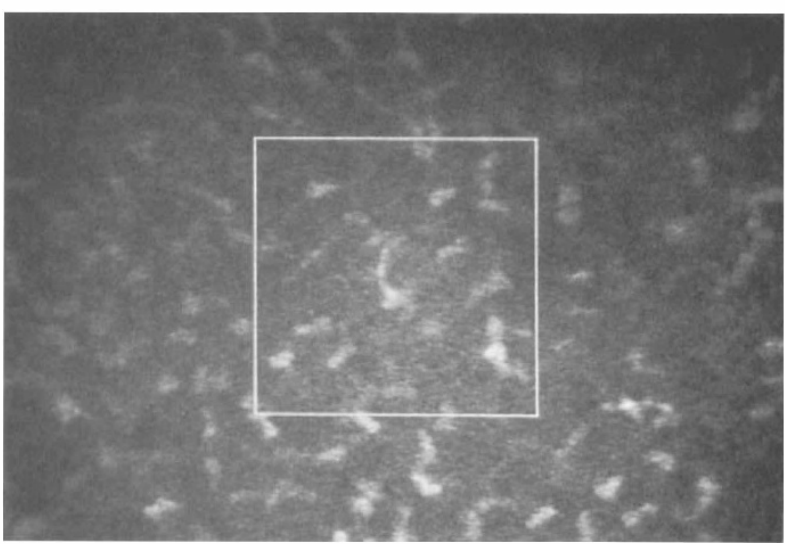

Fig. 1. Grey-scale confocal image of keratocytes in superficial stroma. Single video field digitised to 8 bits, resolution $768 \times 512$. Field of view $630 \mu \mathrm{m} \times 420 \mu \mathrm{m}$. The section marked is that used in image processing, $256 \times 256$ pixels, $210 \times 210 \mu \mathrm{m}$.

However, images were brighter in the centre than at the edge and thus a suitable threshold level in the centre was not appropriate for the entire image, and could incorrectly portray the size and shape of cells. Variations in brightness were due in part to the camera. Also illumination was brighter in the centre. It was possible to adjust a condenser lens to produce more even illumination, but this reduced image contrast.

Even within small sections of images where background intensity was nearly constant, careful manual setting of an appropriate threshold level was necessary to select cell shapes. Several other small bright areas were included in the resultant binary image. It was presumed that these bright spots were not from cells and were considered to be 'noise'.

\section{Procedure for image analysis}

Several approaches were used to overcome these problems. An outline of the procedure is given below, followed by a detailed description.

\section{Outline}

1. Contrast was enhanced. This made the range of brightness levels the same in all images: from 0 to 255 (arbitrary units).

2. There was less variation in background intensity across small areas of the image. Small sections from the centre of images were processed. Images of the background were computed and then subtracted from the recorded image. This resulted in images with more uniform background. They contained cell shapes, but also noise.

3. A threshold brightness level was set at a point between 0 and 255. Pixels less than the threshold were set to zero, those greater to a value 1 . This gave a binary image.

4. Binary images contained cell shapes but also 'noise' (not cell structure). 'Noise' was removed from images. Groups of pixels that were too small to be cells, or 
were of a different shape from cells, were removed. This gave images in which the background was at value zero and cell structures at value 1 .

5. Such images could then be processed to give information about cell structure.

\section{Details}

1. Contrast enhancement Contrast was enhanced by the process of 'normalisation'. 9 A new value for each pixel of an image was determined using the equation

$$
P^{\prime}=255 \times\left[\frac{(P-\min )}{(\max -\min )}\right]
$$

where $P^{\prime}$ is the new pixel value, $P$ is the original pixel value, max is the value of the brightest pixel in the original image and $\mathrm{min}$ is the value of the darkest pixel in the original image.

2. Subtraction of background Processing was done using only the central region of images, $256 \times 256$ pixels, as within this area there was less variation in background intensity. Further flattening was achieved by subtracting an image of background alone. It was found necessary to use a background image specific to each subject. Such images were made by averaging all digitised images from a subject. This gave a featureless image without structural detail. However, averaging revealed streaks caused by the scanning holes in the disc. These were removed by a standard image averaging process in which each pixel is replaced by the average of itself and the surrounding eight pixels. ${ }^{9}$ This filter was performed 15 times and resulted in background images of smooth profile, slightly brighter in the centre than at the edge.

3. Thresholding of images A suitable threshold level was then determined. The same level could not be used for all images and therefore the relationship between the appropriate threshold level and various parameters of the image was investigated.

Eleven images from each of three subjects were processed as described above. The best threshold level for each was then chosen 'by eye'. Mean intensity in the image, minimum and maximum frequencies of pixel values, the value at the most frequent intensity and the standard deviation of pixel values were determined for each image. The selected threshold level was correlated with each of these parameters. The highest correlation was found to be with the calculated mean intensity. Results are plotted in Fig. 2.

Linear regression analysis gave:

$$
T=(0.481 \times M)-0.216
$$

where $T$ is the estimated threshold and $M$ is the mean of all pixel intensities in image. The correlation coefficient was 0.92 . At a typical mean intensity $(M=22, T=10)$, the $95 \%$ confidence interval was \pm 1 . This equation was used to determine the threshold level in the automated image analysis process.

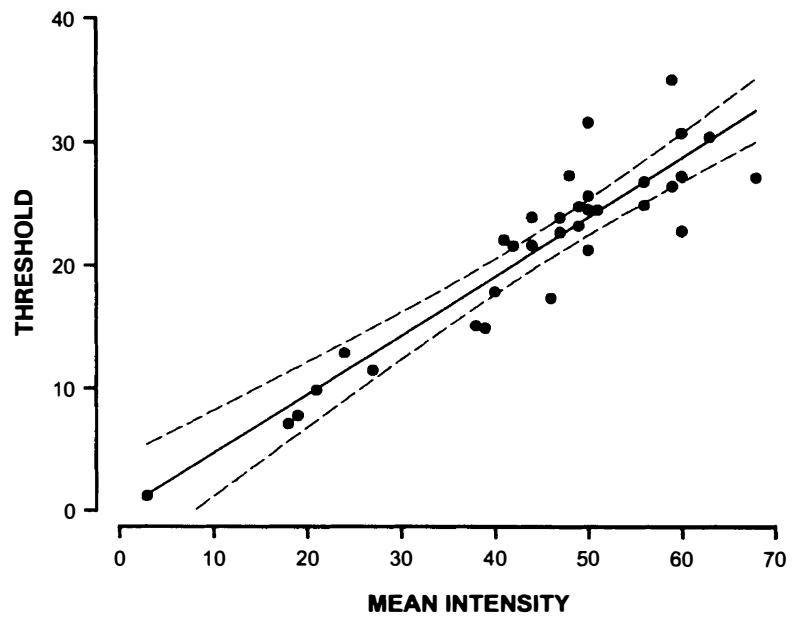

Fig. 2. Correlation of threshold values with mean intensity. The best threshold level for each of 33 sample images, selected 'by eye', is plotted against mean intensity of the image. Units of intensity are arbitrary, range 0-255. The linear regression line is shown; constant -0.216 , coefficient 0.481 , correlation coefficient 0.92 . Dashed lines are $95 \%$ confidence limits.

4. Removal of noise Binary images included all cells, but there were also random small areas that did not seem to be related to cellular structure. These were removed from images by two processes.

The first excluded those objects smaller than 50 pixels in area (approximately $15 \mu \mathrm{m}^{2}$ ). Cells were larger than this (greater than $78 \mu \mathrm{m}^{2}$ ), and small changes in the cutoff did not give substantially different results.

The second process filtered out objects circular in shape. Keratocytes tended to be elongated, sometimes U-shaped, but rarely circular. Thus noise was also removed by excluding circular objects. The parameter $S$, as defined below, was measured for each object in images:

$$
S=\frac{4 \pi a}{p^{2}}
$$

where $S$ is the shape of the object, $A$ is the area of the object and $p$ is the perimeter of the object. For a circular object, $S=1$. For a long thin object, $S$ approaches zero. Various cut-off levels were tested and 0.5 found to be suitable.

The combination of these two filters removed noise from images.

5. Final stages Any holes within objects were filled with pixels of value 1 . These were usually small (1-3 pixels).

Analysis of binary images gave the percentage of the area of images occupied by cells and the number of cells per image. Thus the cross-sectional mean cell size and density of cells per square millimetre could be calculated. If only part of a cell was visible, cut off by the edge of the image, it was counted as one whole cell.

Processing was done on a 486 PC using 'Lucida', an image processing package (Kinetic Imaging, Liverpool, UK). Analysis for each subject required about $12 \mathrm{~h}$, but was automated and ran unattended. 


\section{Image calibration}

Images were calibrated by digitising images of a silicon crystal marked with squares at a periodicity of $9.9 \mu \mathrm{m}$ (Agar Scientific, Stanstead, UK). Although there was pincushion distortion at the edge of images, spacing in the central area of $256 \times 256$ pixels was regular at 1 pixel $=$ $0.833 \mu \mathrm{m}$.

\section{Results}

A sample image is shown in Fig. 1. The optical section was through stroma immediately deep to Bowman's membrane. The central region of $256 \times 256$ pixels used in analysis is shown in Fig. 3a and the processed binary image in Fig. 3b. Optical sections through deep stroma, just superficial to Descemet's membrane, in the same subject are shown in Fig. 3c (grey-scale image) and Fig. 3d (binary image).

Density of cells in each subject is plotted against depth from the epithelial surface in Fig. 4. The percentage of image area occupied by keratocytes is shown in Fig. 5. Cellularity was greatest near the stromal surface and reduced sharply towards posterior cornea except

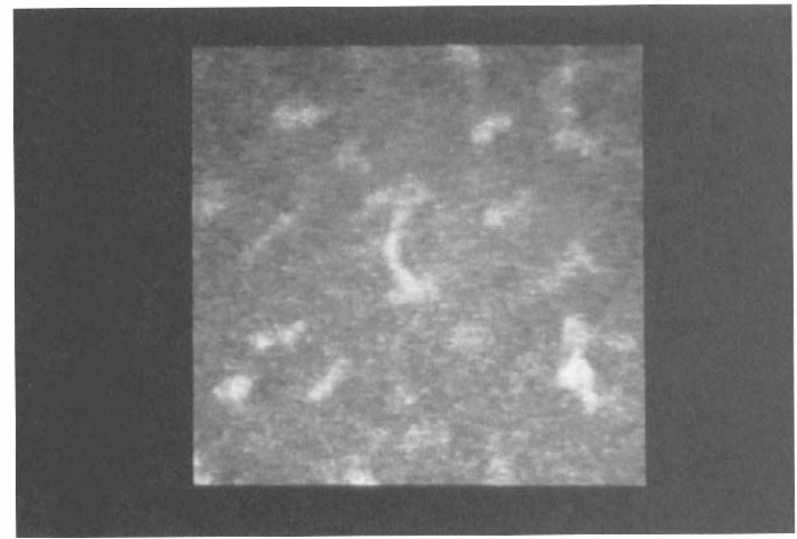

(a)

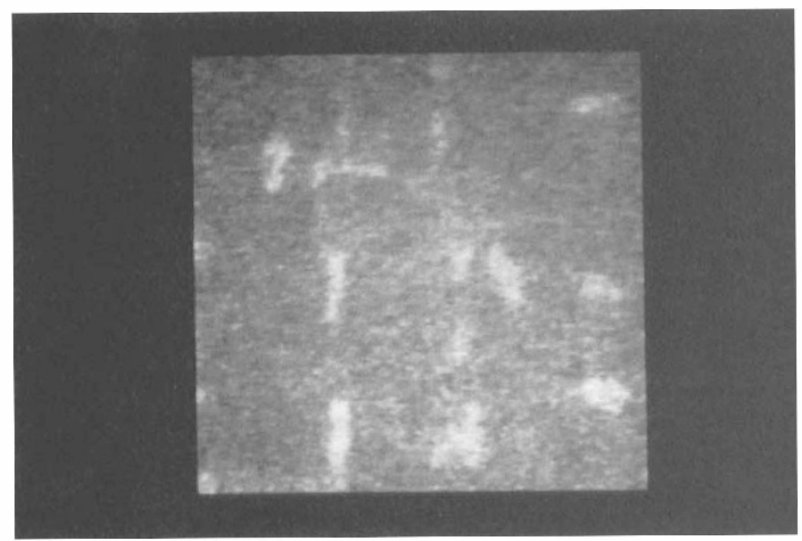

(c) approximately $50 \mu \mathrm{m}$ from the endothelium, where there was an increase in cell density and area. The decrease in values deep to this region may have been in part because optical sections passed into Descemet's membrane. Both plots show a similar pattern through the whole stroma. Thus changes in area seem to be largely a result of changes in density, the size of cells remaining approximately constant. Plots of cell size against depth did not show a consistent trend. Cells in superficial regions were not of a significantly different size to those in mid-stroma or deep stroma. The size of cells varied from 77.7 to $210.6 \mu \mathrm{m}^{2}$, mean $151.1 \mu \mathrm{m}^{2} \pm 29.8$ (standard deviation). Density of cells ranged from 59.0 to $848.1 \mathrm{~mm}^{-2}$, mean $264.9 \mathrm{~mm}^{-2} \pm 152.0$.

There were no cells in Bowman's membrane. Video recordings of scans through the cornea showed a cell-free layer between the subepithelial nerve plexus and most superficial region of stroma that contained keratocytes. This is consistent with the work of Poole et al. ${ }^{4}$ who used fluorescent dyes to examine the distribution of keratocytes and found there to be none within Bowman's membrane.

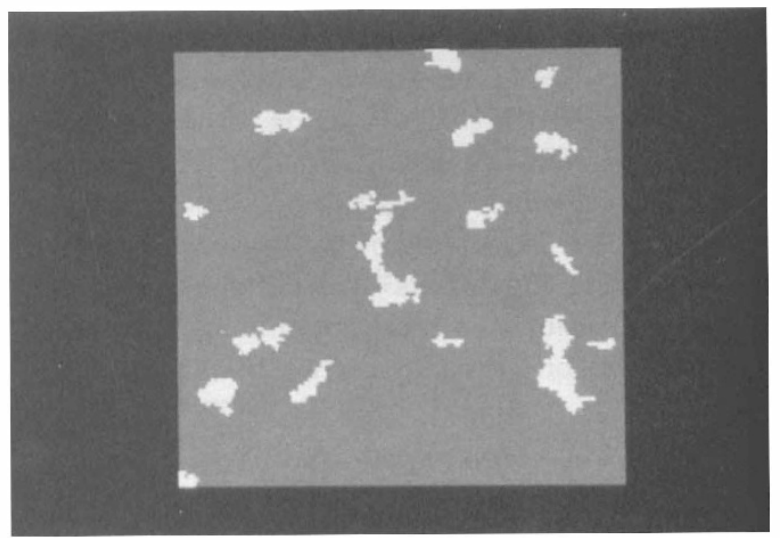

(b)

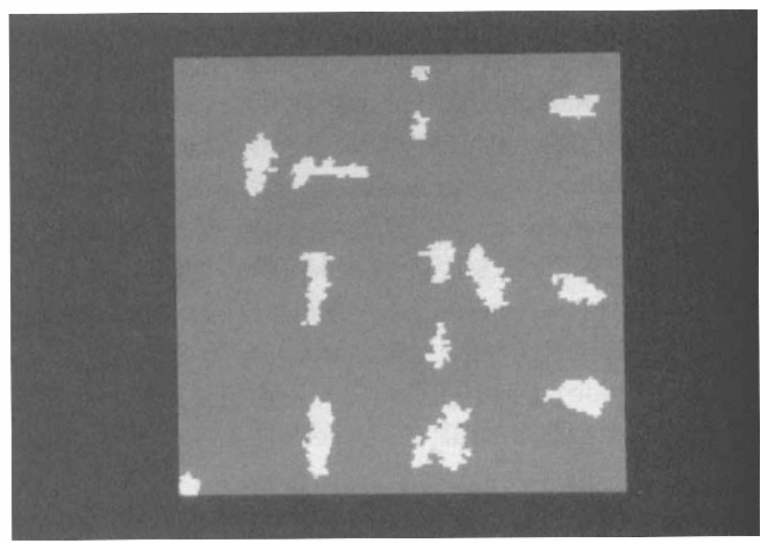

(d)

Fig. 3. Images before and after processing. (a) Grey-scale image of a section through superficial stroma. The full-sized image is shown in Fig. 1. (b) Binary image. Result of processing image shown in (a). Grey areas are of value 0, white of value 1. (c). Grey-scale image. Section $25 \mu$ m superficial to Descemet's membrane. From the same subject as images in (a) and (b). (d) Binary image after processing of image shown in (c). 


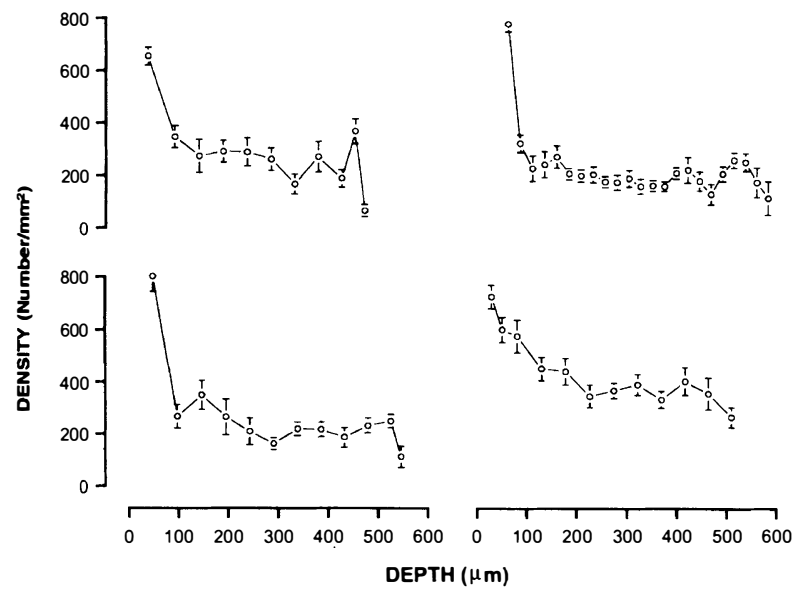

Fig. 4. Density of cells (number $/ \mathrm{mm}^{2}$, mean \pm standard error) plotted against depth of image from epithelial surface in each of four subjects.

\section{Discussion}

The methods used give measurements of keratocyte density, the area of images occupied by them and their size. Confocal microscopy is sensitive to small changes in refractive index within tissues and, as a result, fixation and staining is not required to reveal microscopical detail. Also, the resolving power and image quality are much enhanced by the narrow field examined at one time, free from blurred and scattered light from the surroundings. It is these properties that make it possible to examine functioning living tissue. The images are quite different from those produced by light, fluorescence or electron microscopy - each of which requires complex procedures to prepare specimens and enable imaging of internal structure. The preparation and processing of specimens may introduce artefacts. During examination by confocal microscopy there is no physical or chemical manipulation of the tissue and it is possible that artefacts of this kind are eliminated. On the other hand, confocal imaging may have problems and introduce distortions of its own. For instance, digital processing of binary reduced data may introduce its own

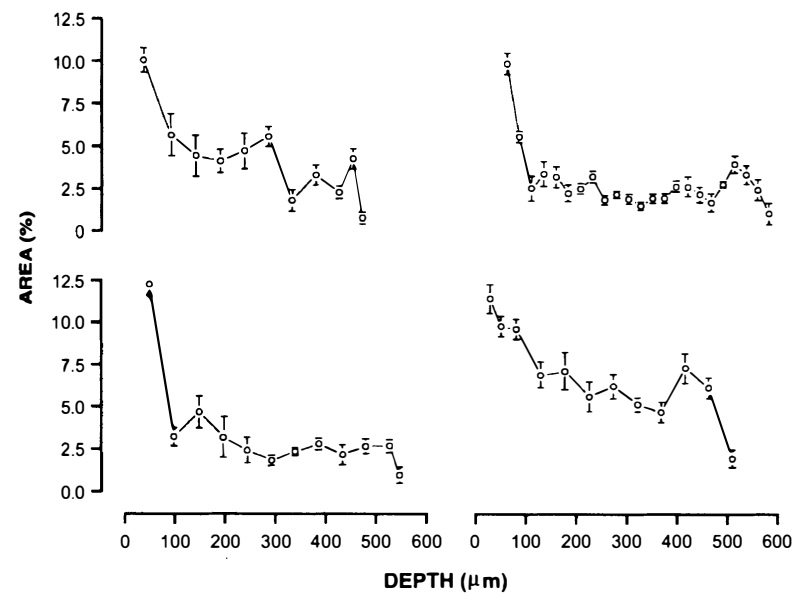

Fig. 5. Percentage area of images occupied by cells plotted against depth of image from epithelial surface in each of four subjects (mean \pm standard error). particular factors. As a result, the number, size and shape of cells imaged by the confocal microscope may be determined in some way by its properties. However, this consideration is in no way special to this new form of microscopy, but applies equally to all techniques for imaging biological material.

It is relevant to consider whether our methods in some way manufactured artificial results. Processing involved selection of various parameters to enable quantification. Objects smaller than $15 \mu \mathrm{m}^{2}$ were excluded. However, the minimum size of cell in all subjects was much larger: $78 \mu \mathrm{m}^{2}$. It is possible that smaller cellular structures, perhaps parts of cells cut off by optical sectioning, were excluded from analysis. Thus some structural detail may have been removed. The filtering process was necessary to give a closer match between processed images and original video recordings. It removed only a small proportion of objects. Tests indicated that measurements of cell density would have been about $1 \%$ larger if the filter had not been used.

Measurements of the density of cells are given in units per unit area. Reconstruction of their three-dimensional structure to enable measurement of density per unit volume and of cell volume was not done. Constant involuntary eye movements resulted in misalignment of structures in consecutive slices through the cornea and as a result the necessary reconstruction was not possible. Cell size and density per unit area may not correspond to the three-dimensional arrangement if the shape or orientation of cells varies significantly at different depths in the cornea.

Comparison of binary and grey-scale images indicated that processing gave a close representation of cells in the original image, as shown in Fig. 3. Changes in measurements through the stroma were as anticipated from reviewing video recordings and closely follow manual counts by eye. However, when counting by eye it is difficult to maintain fixed criteria and reproducibility is poor. One of the aims of developing the methodology presented in this paper was to eliminate observer error.

It is possible that cells at different depths in the cornea reflect light differentially, perhaps resulting in fewer cells being seen at deeper sections through the cornea. However, cell density and size did not decrease progressively through the stroma. Also, our results are consistent with earlier work using histological and biochemical techniques in isolated tissues. These are discussed below. Thus it seems that the results presented here are a close measure of cell structure in confocal images.

Møller-Pedersen et al. ${ }^{5}$ determined the density of keratocytes in human post-mortem corneas using conventional microscopy and found a good correlation with measurements of tissue DNA content. Cell density measured $100-350 \mathrm{~mm}^{-2}$. It did not vary with the length of time tissue was stored in culture medium and the authors took this to indicate that measurements were similar to those in living tissue. We found values of up to 
$800 \mathrm{~mm}^{-2}$ in superficial optical sections, but deep to this layer cell density was close to the range found in their work.

Poole et al., ${ }^{4}$ using fluorescent dyes in pig and steer cornea, found a high concentration of cells in a narrow band deep to Bowman's membrane, little variation through most of the stroma, and increased density immediately superficial to Descemet's membrane. In this region they reported cells to be larger and more irregularly shaped than those in the mid-stroma. Cell density was found by Boettcher et al. ${ }^{10}$ to decrease from superficial to deep stroma in rabbit cornea, with an increase just before the endothelium. Similar results were obtained by Møller-Pedersen and Ehlers ${ }^{6}$ using DNA content to calculate cell density in human cornea. However, they found the decrease in cell density to be limited to the posterior half of the cornea. An increase just superficial to endothelium was not reported.

Thus our techniques give results that are consistent with earlier findings in isolated tissue, but provide a method for examining cell morphology in conscious human subjects. The ability to automate analysis is important because of the large number of images generated by confocal recordings. The methods we have developed enable studies of ongoing processes in living tissue.

\section{References}

1. Cavanagh HD, Petroll WM, Alizadeh H, Yu-Guang H, McCulley JP, Jester JV. Clinical and diagnostic use of in vivo confocal microscopy in patients with corneal disease. Ophthalmology 1993;100:1444-54.

2. Klyce SD, Beuerman RW. Structure and function of the cornea. In: Kaufman HE, Barron BA, McDonald SR. The cornea. Edinburgh: Churchill Livingstone, 1988:3-54.

3. Katakami C, Sahori A, Kazusa R, Yamamoto M. Keratocyte activity in wound healing after epikeratophakia in rabbits. Invest Ophthalmol Vis Sci 1991;32:1837-45.

4. Poole CA, Brookes NH, Clover GM. Keratocyte networks visualised in the living cornea using vital dyes. J Cell Sci 1993;106:685-92.

5. Møller-Pedersen T, Ledet T, Ehlers N. The keratocyte density of human corneas. Curr Eye Res 1994;13:163-9.

6. Møller-Pedersen T, Ehlers N. A three-dimensional study of the human corneal keratocyte density. Curr Eye Res 1995;14:459-64.

7. Petroll WM, Boettcher K, Barry P, Cavanagh HD, Jester JV. Quantitative assessment of anteroposterior keratocyte density in the normal rabbit cornea. Cornea 1995;14:3-9.

8. Petroll WM, Cavanagh HD, Jester JV. Three-dimensional imaging of corneal cells using in vivo confocal microscopy. J Microsc 1993;170:213-9.

9. Gonzalez RC, Wintz P. Image enhancement. In: Digital image processing, 6th ed. Reading, Mass: Addison-Wesley, 1983:118-54.

10. Boettcher K, Petroll WM, Jester JV, Cavanagh HD. Determination of 3-dimensional keratocyte density in the rabbit cornea using laser confocal microscopy. Invest Ophthalmol Vis Sci 1994;35:1355. 